\title{
Photoacoustic investigation of semiconductors: Influence of carrier diffusion and recombination in $\mathrm{PbTe}$ and $\mathrm{Si}$
}

\author{
A. Pinto Neto and H. Vargas \\ Instituto de Física, Universidade Estadual de Campinas, 13100-Campinas, SP, Brazil \\ N. F. Leite and L. C. M. Miranda \\ Laboratório Associado de Sensores e Materiais, Instituto de Pesquisas Espaciais, Caixa Postal 515, \\ 12201-São José dos Campos, SP, Brazil
}

(Received 12 December 1988)

\begin{abstract}
The photoacoustic signal of a narrow-gap semiconductor and of $\mathrm{Si}$ is investigated as a function of the modulation frequency through the use of a heat-transmission configuration. It is shown that in the thermally thick modulation-frequency range the signal amplitude can single out the different heating sources responsible for the photoacoustic signal. It is also shown that from the signal phase data, as a function of the modulation frequency, we can obtain the values of the surface recombination velocity and the nonradiative band-to-band recombination time.
\end{abstract}

\section{INTRODUCTION}

The photoacoustic (PA) and related photothermal techniques has proved to be a valuable tool in the semiconductor research field. This is demonstrated by the increasing number of publications ${ }^{1-7}$ in this area in the last ten years. For a review on the subject of photoacoustics we refer to the work of Ref. 8 whereas for a comprehensive discussion on the PA applications to semiconductor research we refer to the book by Mandelis. ${ }^{9}$

The PA signal is directly related to the amount of light absorbed by the sample and converted into heat through nonradiative deexcitation processes. In the case of semiconductors, the heat source resulting from the absorption of light depends not only on the optical properties of the sample itself but also on their transport (e.g., carrier lifetime, diffusion length, surface recombination velocity) and nonradiative state properties. Physically, this may be seen as follows. The absorption of light generates excess carrier distribution in the sample. These excess carriers diffuse through the sample and reestablish equilibrium by disposing of the energy in excess both by emitting radiation and by generation of heat. The PA technique responds only to the fraction of this energy that is converted into heat. The heat generated in this process of reestablishing equilibrium is essentially due to intraband transitions in the bulk, nonradiative intraband transitions, and nonradiative surface recombinations. Apart from this, the PA effect in semiconductors is also sensitive to the presence of nonradiative states near the surface of the sample as demonstrated by several authors. The influence of the carrier lifetime, diffusion coefficient, and nonradiative transitions in the PA signal of semiconductors was first demonstrated by Ghizoni and coworkers $^{3}$ and by Mikoshiba et al. ${ }^{4}$ in the case of piezoelectric detection.

In this paper we investigate the PA signal of a narrowgap semiconductor such as $\mathrm{PbTe}$ and of $\mathrm{Si}$ and show that by recording both the PA signal amplitude and phase, as a function of the modulation frequency, one can readily obtain the nonradiative carrier lifetime and surface recombination velocity. These physical parameters are usually obtained from photoconductivity measurements. ${ }^{10}$ However, for narrow-gap semiconductors, due to the large carrier concentration at room temperature, the photoconductivity measurements lead to no information on these parameters. The PA technique is shown to be an alternative simple method for measuring these parameters at room temperature.

\section{EXPERIMENTAL RESULTS}

The experiments were performed using either the socalled open-photoacoustic cell ${ }^{11,12}$ (OPC) or the conventional PA transmission cell. ${ }^{13}$ The OPC apparatus is schematically shown in Fig. 1(a). The light source consists of a $250-\mathrm{W}$ W lamp, which after being mechanically chopped is focused onto the sample. To ensure no effective dc heating of the samples and that all the light absorption is taking place at the rear surface of the samples, the white light was filtered for wavelengths greater than $800 \mathrm{~nm}$ using a heat filter. The samples were mounted directly onto the front sound inlet of an electret microphone (EM-60 from Primo Co.). The sound inlet is a circular hole of $3 \mathrm{~mm}$ diameter, and the front microphone air chamber adjacent to the metallized face of the diaphragm is a cylinder $7 \mathrm{~mm}$ in diameter and roughly 1 mm long. The conventional PA transmission measurements ${ }^{13}$ were performed in a brass cell in which a quarter-inch condenser microphone (Brüel and Kjaer) is mounted in one of its walls. The samples were flush against the back wall of the cell which has a 4-mm-diam hole allowing the PA transmission measurements to be carried out, as shown in Fig. 1(b).

The samples used in our measurements were $p$-type (100) single crystals of PbTe and $\mathrm{Si}$. The PbTe sample 
(a)

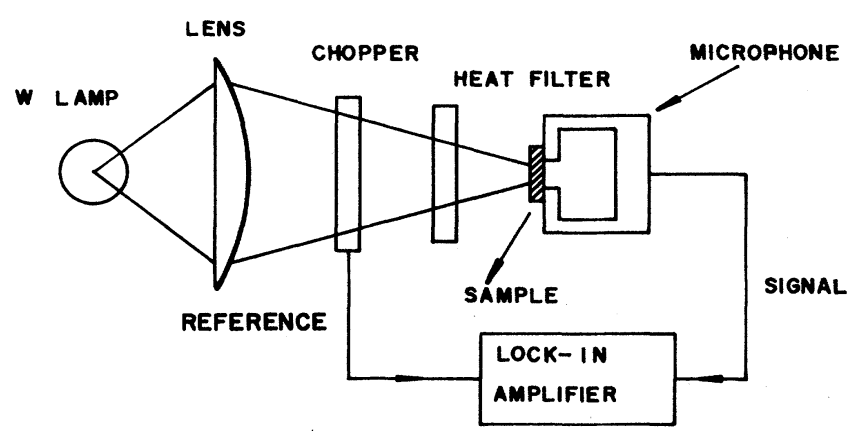

(b)

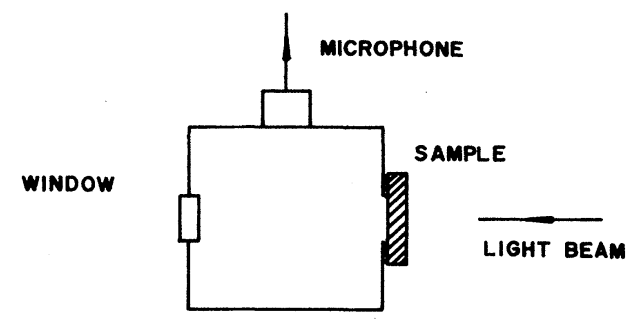

FIG. 1. (a) Experimental arrangement used in the OPC measurements, and (b) in the conventional PA transmission configuration.

was grown by the vapor-melt-solid method, as discussed in Refs. 14-16. The room-temperature measured values of the relevant transport and electrical properties of the samples are listed in Table $I$. The carrier diffusion coefficient $D$ was calculated from the Hall mobility measurements, using the Einstein relation, namely, $D=\mu k_{B} T / e$. To investigate the influence of the surface recombination velocity on the PA signal, the sample surface in contact with the air of the PA chamber had different finishings, whereas the (back)heating surface always had the same finish. One finish, the so-called polished surface (PS), was a mirrorlike etching. In the case of the $\mathrm{PbTe}$ sample the polished surface finish was obtained by a sequence of mechanical polishing using $\mathrm{Al}_{2} \mathrm{O}_{3}$ powder of different meshes $(9,5$, and $2 \mu \mathrm{m})$ followed by a

TABLE I. Electrical and transport properties of $p$-type PbTe and Si samples, as obtained from the Hall measurements.

\begin{tabular}{lll}
\hline Physical parameters & \multicolumn{1}{c}{ PbTe } & \multicolumn{1}{c}{$\mathrm{Si}$} \\
\hline Mobility $\left(\mathrm{cm}^{2} / \mathrm{V} \mathrm{s}\right)$ & 925 & 431 \\
Resistivity $(\Omega \mathrm{cm})$ & 0.01 & 58.4 \\
$\begin{array}{l}\text { Carrier } \\
\quad \text { concentration }\left(\mathrm{cm}^{-3}\right)\end{array}$ & $6.8 \times 10^{17}$ & $2.5 \times 10^{14}$ \\
$\begin{array}{l}\text { Carrier } \\
\quad \text { coefficient }\left(\mathrm{cm}^{2} / \mathrm{s}\right)\end{array}$ & 23.96 & 11.16 \\
$\begin{array}{l}\text { Thermal } \\
\text { diffusivity }\left(\mathrm{cm}^{2} / \mathrm{s}\right)\end{array}$ & 0.015 & 1.06 \\
\hline \hline
\end{tabular}

chemical etching using an iodine-methanol solution. The Si sample was already mirrorlike polished from the manufacturers. The roughened surface (RS) finish was obtained by simply polishing the sample surface with 9$\mu$ m-mesh $\mathrm{Al}_{2} \mathrm{O}_{3}$ powder. The (back)heating surface of both samples always had a roughened finish. In what follows, we shall denote simply by RS and PS the samples for which the surface in contact with the air of the PA chamber is roughened and polished, respectively.

In Figs. 2 and 3 we show the OPC signal amplitude of the PbTe sample as a function of the modulation frequency for the case of a $298-\mu \mathrm{m}$-thick RS sample and a 320$\mu$ m-thick PS sample, respectively, whereas in Fig. 4 we show the PA signal amplitude for a $390-\mu$ m-thick PS Si sample illuminated by a $150-\mathrm{mW} \mathrm{Ar}^{+}$laser, using the conventional PA transmission configuration. The solid lines in all these figures represent the best fit to the data, either a power law, $f^{-v}$, or an exponential law of the form $\exp (-a \sqrt{f})$. This fitting procedure led, for the case of the RS PbTe sample shown in Fig. 2, to the following results. Between 50 and $70 \mathrm{~Hz}$ the signal amplitude data is best fitted by an exponential law [namely, $s \sim \exp (-a \sqrt{f})]$, whereas between 80 and $120 \mathrm{~Hz}$ the signal varies as $f^{-1.55}$, and, finally, between 135 and 200 $\mathrm{Hz}$ the PA signal behaves as $f^{-0.92}$. For the case of the PS PbTe sample shown in Fig. 3, the PA signal is best fitted in the 50 to $70 \mathrm{~Hz}$ region by an exponential law too; between 70 and $105 \mathrm{~Hz}$ the signal varies as $f^{-1.6}$, and from 120 to $200 \mathrm{~Hz}$ it is best fitted by a power law of the form $f^{-0.89}$. In contrast, the data for the PS Si sample is best fitted, in the whole frequency range of our measurements, by a power law of the form $f^{-1.47}$. Using the values of the thermal diffusivity of PbTe (i.e., $\alpha=0.015$ $\mathrm{cm}^{2} / \mathrm{s}$ ) and of $\mathrm{Si}$ (i.e., $\alpha=1.06 \mathrm{~cm}^{2} / \mathrm{s}$ ) we find that the threshold modulation frequencies for the transition into the thermally thick regime for our samples are $5.4 \mathrm{~Hz}$ for the RS PbTe sample, 4.7 $\mathrm{Hz}$ for the PS PbTe sample, and

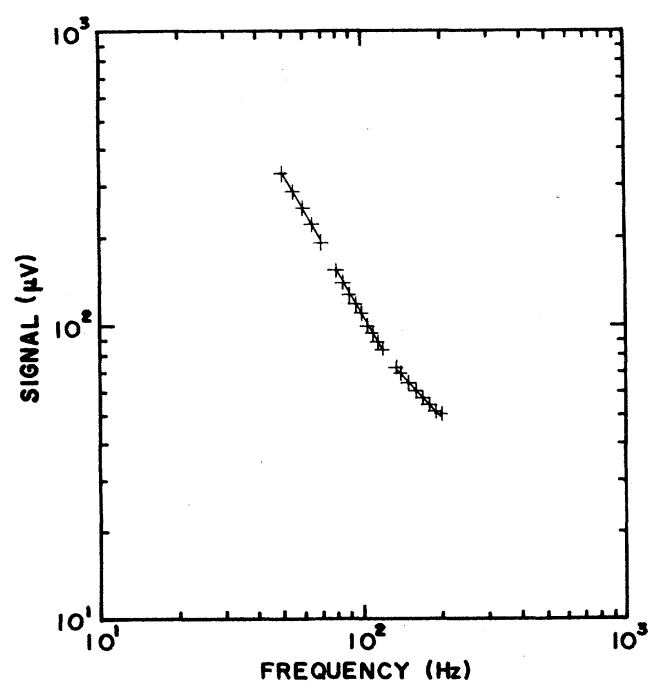

FIG. 2. OPC signal amplitude for the $298-\mu \mathrm{m}$-thick roughened surface $\mathrm{PbTe}$ sample as a function of the modulation frequency. 


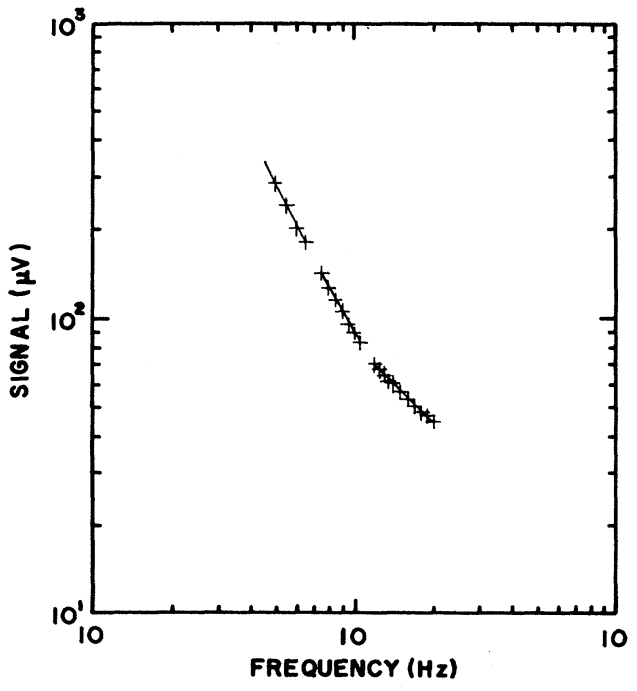

FIG. 3. OPC signal amplitude for the $320-\mu \mathrm{m}$-thick polished surface $\mathrm{PbTe}$ sample as a function of the modulation frequency.

$222 \mathrm{~Hz}$ for the Si sample. Thus, in the 50 to $200 \mathrm{~Hz}$ modulation-frequency range of our measurements, the $\mathrm{PbTe}$ samples are both deep into the thermally thick regime, whereas the Si sample is in the thermally thick regime above roughly $220 \mathrm{~Hz}$. The simple thermal diffusion model with an instantaneous heat source of Rosencwaig and Gersho ${ }^{17}$ (RG) predicts that, for a thermally thick sample, the PA signal in the transmission configuration should vary exponentially as $\exp (-a \sqrt{f})$, where $a=l(\pi / \alpha)^{1 / 2}, l$ is the sample thickness, and $\alpha$ is the thermal diffusivity. The data for the thermally thick PbTe samples shown in Figs. 2 and 3 exhibited this exponential behavior only up to roughly $70 \mathrm{~Hz}$.

To decide whether the exponential behavior was the best fit to the data, we have compared it with the powerlaw fitting, and looked at which one yielded the smallest error. For example, for the RS PbTe sample the error in the exponential fitting was $0.32 \%$, whereas the power-law fitting yielded an error of $1.8 \%$. In fact, using the values of the parameter $a$, obtained from the data fitting for each PbTe sample, namely, $a=0.415$ and $a=0.48$ for the RS and PS samples, respectively, we have estimated their thermal diffusivity to be $\alpha=0.016$ and $0.014 \mathrm{~cm}^{2} / \mathrm{s}$, respectively. These values are in very close agreement with the literature value of $\mathrm{PbTe}$, namely, $\alpha=0.015 \mathrm{~cm}^{2} / \mathrm{s}$, thereby confirming that the exponential law is the best fit to the data in this frequency region. Above $80 \mathrm{~Hz}$, however, both samples exhibited a power-law dependence on the modulation frequency, indicating that, deep into the thermally thick regime, the PA signal is not described by the thermal diffusion model with an instantaneous heat source. For the Si sample, even in the beginning of the thermally thick regime, the PA signal did not exhibit the exponential behavior, as shown in Fig. 4. The understanding of this power-law behavior of the PA signal in the thermally thick regime is the problem to which we address ourselves in the following.

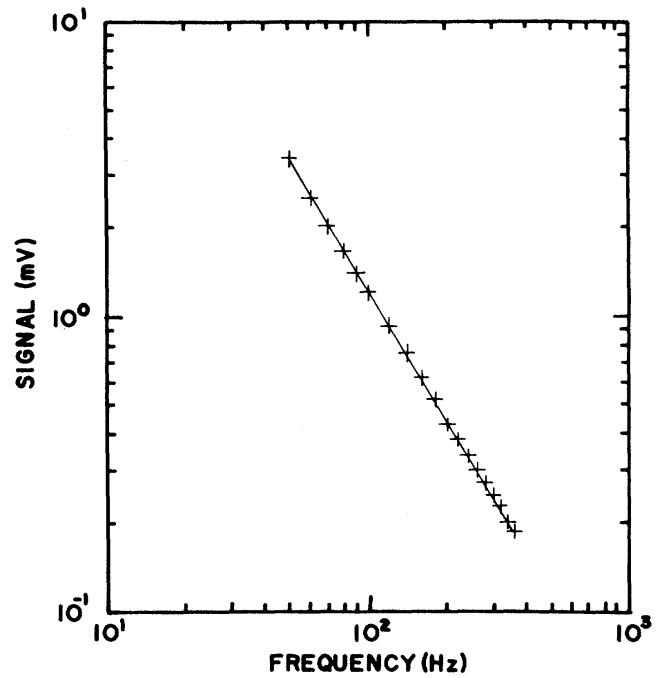

FIG. 4. PA signal amplitude for the $390-\mu \mathrm{m}$-thick polished surface Si sample, illuminated by a $150-\mathrm{mW} \mathrm{Ar}^{+}$laser, as a function of the modulation frequency, using the conventional PA cell.

\section{DISCUSSION}

Consider the PA cell geometry for the heattransmission configuration shown schematically in Fig. 5. According to the thermal-piston model of $R G,{ }^{17}$ the pressure fluctuation $\delta P$ in the gas region, due to the periodic heating of the sample, is given by

$$
\delta P=\frac{P_{0} \Theta}{T_{0} l_{g} \sigma_{g}} e^{j \omega t},
$$

where $P_{0}\left(T_{0}\right)$ is the ambient pressure (temperature), $l_{g}$ is the length of the gas chamber, $\sigma_{g}=(1+j) a_{g}$, $a_{g}=\left(\pi f / \alpha_{g}\right)^{1 / 2}$ is the thermal diffusion coefficient in the gas with thermal diffusivity $\alpha_{g}$, and $\Theta$ is the sample temperature fluctuation at the $x=0$ sample-gas interface. The temperature fluctuation is obtained from the solution to the thermal diffusion equation, namely,

$$
\frac{\partial^{2} T}{\partial x}=\frac{1}{\alpha_{s}} \frac{\partial T}{\partial t}-\frac{Q(x, t)}{k_{s}},
$$

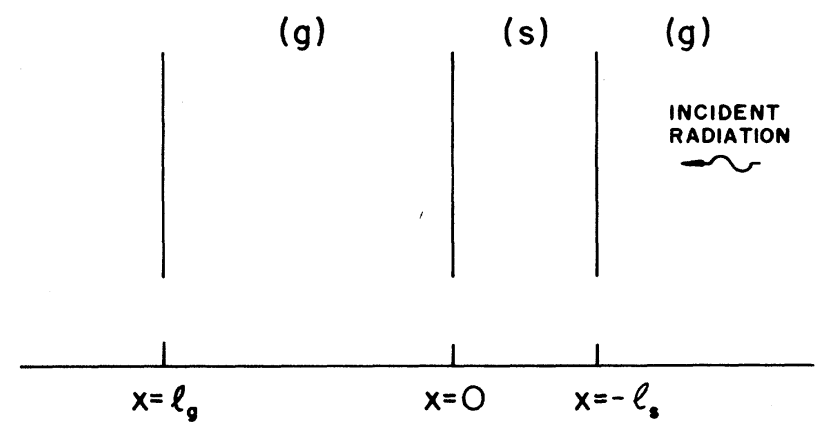

FIG. 5. PA cell geometry for the heat-transmission configuration. 
where $\alpha_{s}\left(k_{s}\right)$ is the sample thermal diffusivity (conductivity), and $Q(x, t)$ is the heat power density generated in the sample due to the absorption of light. In the case of semiconductors, a proper description of the sample heating should take into account the spatial and temporal dispersion effects due to carrier diffusion, as follows. The absorption of photons with energy greater than the gap energy $E_{g}$ creates electron-hole pairs. These carriers diffuse through the sample and recombine nonradiatively both in the bulk and at the surface, thereby generating the thermal wave. Thus, the thermal power density $Q(x, t)$ may be regarded as due to the following three different processes. ${ }^{18}$ (i) An instantaneous intraband nonradiative thermalization with energy greater than $E_{g}$. This process, due to the electron-phonon collisions within the conduction band, occurs on a time scale of ns and may be assumed instantaneous in the typical range of modulation frequencies of photoacoustics. The heat power density due to this process, denoted by $Q_{D}$, is then

$$
Q_{D}=\frac{\beta\left(h v-E_{g}\right)}{h v} I_{0} e^{\beta\left(x+l_{s}\right)} e^{j \omega t},
$$

where $\beta$ is the optical absorption coefficient for photons of energy $h v$, incident at the $x=l_{s}$ surface with an intensity of $I_{0} \mathrm{~W} / \mathrm{cm}^{2}$. (ii) Nonradiative bulk recombination. This process is due to the excess electron-hole pairs recombination after diffusing a distance $(D \tau)^{1 / 2}$, where $D$ is the carrier diffusion coefficient, and $\tau$ is the band-toband recombination time. The heat power density $Q_{\text {NRR }}$ for this process is

$$
Q_{\mathrm{NRR}}=\frac{E_{g}}{\tau} n(x, t),
$$

where $n(x, t)$ is the density of photoexcited excess carriers. (iii) Nonradiative surface recombination. The heat power density $Q_{\mathrm{SR}}$ due to the nonradiative carrier recombination at the sample surface is written as

$$
Q_{\mathrm{SR}}=E_{g}\left[v \delta(x)+v_{0} \delta\left(x+l_{s}\right)\right] n(x, t),
$$

where $v$ is the carrier surface recombination velocity at the back (heating) surface, and $v$ is the surface recombination velocity at the sample-gas interface at $x=0$.

It follows from the above that the solution to Eq. (2) depends explicitly on $n(x, t)$, which, in turn, obeys the carrier diffusion equation, namely,

$$
\begin{aligned}
\frac{\partial n}{\partial t}= & D \frac{\partial^{2} n}{\partial x^{2}}-\frac{n}{\tau}+\frac{\beta I_{0}}{h v} e^{\beta\left(x+l_{s}\right)} e^{j \omega t}-v n(x, t) \delta(x) \\
& -v_{0} n\left(-l_{s}, t\right) \delta\left(x+l_{s}\right) .
\end{aligned}
$$

For the case of short-wavelength incident radiation, as in the case of our experiments, we may assume that all the incident radiation is being absorbed at the $x=-l_{s}$ surface; this corresponds to formally replacing the term $\beta I_{0} e^{\beta\left(x+l_{s}\right)}$ by $I_{0}\left(x+l_{s}\right)$ in Eqs. (3) and (6). Making this assumption and neglecting the heat flux into the surrounding gas, the solution to the coupled equations (2) and (6) leads to the following expression for the sample temperature fluctuation at $x=0$ :

$$
\Theta=\Theta_{D}+\Theta_{\mathrm{NRR}}+\Theta_{\mathrm{SR}}
$$

where

$$
\begin{aligned}
& \Theta_{D}=\frac{2(\epsilon-1) I_{0}}{k_{s} \sigma_{s}\left(e^{l_{s} \sigma_{s}}-e^{-l_{s} \sigma_{s}}\right)}, \\
& \Theta_{\mathrm{NRR}}=\frac{2 \epsilon I_{0}}{k_{s} \sigma_{s}} \frac{\sigma_{s} F}{D \gamma \tau\left(\sigma_{s}^{2}-\gamma^{2}\right)}, \\
& \Theta_{\mathrm{SR}}=\frac{2 \epsilon I_{0} F_{v}}{k_{s} \sigma_{s}\left(e^{l_{s} \sigma_{s}}-e^{-l_{s} \sigma_{s}}\right) D \gamma}\left[\left[1+\frac{1}{D \tau\left(\sigma_{s}^{2}-\gamma^{2}\right)}\right]\left(e^{l_{s} \sigma_{s}}+e^{-l_{s} \sigma_{s}}\right)+\frac{v_{0}}{v}\left[b_{0}(1-r) e^{-\gamma l_{s}}+c_{0}(1+r) e^{\gamma l_{s}}\right],\right.
\end{aligned}
$$

where $\sigma_{s}=(1+j) a_{s}, \quad a_{s}=\left(\pi f / \alpha_{s}\right)^{1 / 2}$ is the thermal diffusion coefficient of the sample, $\gamma=(1 / D \tau)^{1 / 2}(1$ $+j \omega \tau)^{1 / 2}$ is the carrier diffusion coefficient, $\epsilon=E_{g} / h v$, $r=v / D \gamma, r_{0}=v_{0} / D \gamma$, and

$$
\begin{aligned}
& b_{0}=1+\frac{\gamma}{v_{0} \tau\left(\sigma_{s}^{2}-\gamma^{2}\right)}, \quad c_{0}=1-\frac{\gamma}{v_{0} \tau\left(\sigma_{s}^{2}-\gamma^{2}\right)}, \\
& F=\frac{1}{\left(1+r_{0}\right)(1+r) e^{\gamma l}-(1-r)\left(1-r_{0}\right) e^{-\gamma l}} .
\end{aligned}
$$

The neglect of the heat flux into the surrounding gas, as implied in Eqs. (7)-(9), is justified by the fact that the air thermal conductivity is much smaller than that of most materials. This is a widely used approximation in the photothermal theories and corresponds to neglecting the factor $g=\left(k_{g} / k_{s}\right)\left(\alpha_{s} / \alpha_{g}\right)^{1 / 2}$ of the RG model ${ }^{17}$ as compared to 1 . In fact, for $\mathrm{PbTe}$ and $\mathrm{Si}, g$ is equal to $3.74 \times 10^{-3}$ and $3.6 \times 10^{-4}$, respectively, which are negligible as compared to 1 . For $\tau<100 \mu \mathrm{s}$, as is usually the case for most semiconductors at room temperature, and for modulation frequencies smaller than $500 \mathrm{~Hz}$, the product $\omega \tau$ is much smaller than unity so that we may approximate $\gamma$ as $\gamma \simeq(D \tau)^{-1 / 2}$. Under these conditions the parameters $r, r_{0}$, and $F$ become all real constants independent of the modulation frequency. Furthermore, in the case of thermally thick samples (i.e., $l_{s} s_{s} \gg 1$ ), which corresponds to the experimental situation dealt with in 
this paper, the terms involving $\exp \left(l_{s} \sigma_{s}\right)$ are the dominant ones in Eqs. (8a) and (8c). Using these approximations in Eqs. (7) and (8), the pressure fluctuation in the PA cell for a thermally thick sample reduces to

$$
\begin{aligned}
\delta P=\frac{2 \epsilon I_{0} P_{0}}{T_{0} l_{g} \sigma_{g} k_{s} \sigma_{s}}[ & \frac{\epsilon-1}{\epsilon} e^{-l_{s} \sigma_{s}} \\
& \left.+\frac{F \sigma_{s}}{D \gamma \tau}\left[\frac{1}{\sigma_{s}^{2}-\gamma^{2}}+\frac{v \tau}{\sigma_{s}}\right]\right] .
\end{aligned}
$$

The first term in Eq. (10), scaling exponentially with the modulation frequency as $(1 / f) \exp (-a \sqrt{f})$, where $a=l_{s}\left(\pi / \alpha_{s}\right)^{1 / 2}$, is the usual thermal diffusion contribution from an instantaneous heat source, as described by the RG model. The second term in the large square brackets of Eq. (10), which in the high-frequency regime, such that $\sigma_{s}^{2}>\gamma^{2}$, scales as $f^{-1.5}$, is the contribution from the nonradiative bulk recombination. Finally, the last term in Eq. (10) varying as $f^{-1.0}$ is the contribution from the nonradiative surface recombination. Thus, the overall modulation-frequency dependence of the PA signal for a thermally thick semiconductor in the heattransmission configuration is such that it initially varies exponentially as $\exp (-a \sqrt{f})$, as in the usual case of an instantaneous heat source. As we move deep into the thermally thick regime, the instantaneous heat source weakens exponentially and the PA signal becomes dominant by the nonradiative (slow) bulk and surface recombination processes. In this regime, the PA signal varies as $f^{-1.5}$, due to the bulk nonradiative recombination processes, and at higher modulation frequency it scales as $f^{-1.0}$, due to the surface recombination contribution. The above discussion seems to explain the observed PA signal modulation-frequency dependence for both $\mathrm{PbTe}$ and Si samples shown in Figs. 2-4. In particular, the three different modulation-frequency dependences, predicted for the PA signal of a thermally thick sample, are clearly shown in Figs. 2 and 3 for the case of PbTe. In the case of the $\mathrm{Si}$ sample in the thermally thick region, the PA signal remained with the $f^{-1.5}$ dependence exhibiting no $f^{-1.0}$ behavior. We attribute this to the fact that the Si sample had a quite small surface recombination velocity. In fact, even for a RS Si sample we did not observe the $f^{-1.0}$ behavior in the frequency range of our measurements.

Finally, we have attempted to use the above model to obtain the values of $v$ and $\tau$ from our PA transmission data. This was accomplished as follows. In the region where the nonradiative recombination processes are the dominant ones (i.e., when the PA signal amplitude varies as $f^{-v}$ with $v=1.5$ and 1.0 ), Eq. (10) reduces to

$$
\delta P=\frac{2 \epsilon I_{0} P_{0} F}{T_{0} l_{g} k_{s} D \gamma \tau \sigma_{g}}\left(\frac{1}{\sigma_{s}^{2}-\gamma^{2}}+\frac{v \tau}{\sigma_{s}}\right) .
$$

Performing some straightforward but tedious and complex algebra we can show that the phase angle $\phi$ of the PA signal given by Eq. (11) is

$$
\phi=\frac{\pi}{2}+\Delta \phi
$$

where

$$
\tan (\Delta \phi)=\frac{(a D / v)\left(\omega \tau_{\mathrm{eff}}+1\right)}{(a D / v)\left(1-\omega \tau_{\mathrm{eff}}\right)-1-\left(\omega \tau_{\mathrm{eff}}\right)}
$$

with $\tau_{\text {eff }}=\tau\left[\left(D / \alpha_{s}\right)-1\right]$ and $a=\left(\pi f / \alpha_{s}\right)^{1 / 2}$. In Fig. 6 we plot the variable part of the phase angle $\Delta \phi$ as a function of the modulation frequency using the values of $D$ and $\alpha_{s}$ corresponding to a typical PbTe sample, namely, $24 \mathrm{~cm}^{2} / \mathrm{s}$ and $0.015 \mathrm{~cm}^{2} / \mathrm{s}$, respectively, and for $\tau_{\text {eff }}=500$ $\mu \mathrm{s}$. Figure 6 shows that on increasing the modulation frequency the phase angle initially decreases, reaches a minimum, and then increases on further increasing the modulation frequency. The breaking frequency at which $\Delta \phi$ exhibits a minimum corresponds roughly to the point at which the signal changes from the $f^{-1.5}$ to the $f^{-1.0}$ behavior. That is, it marks the transition from the bulk to the surface recombination process as being the dominant mechanism responsible for the PA signal. Furthermore, this transition exhibits an almost linear dependence on the surface recombination velocity. For the example shown in Fig. 6, on doubling the surface recombination, the breaking frequency changed from roughly 45 to 80 Hz. In Fig. 7 we show the variation of the breaking frequency $f_{b}$ as a function of the surface recombination velocity for several values of $\tau_{\text {eff }}$. It follows from this figure that for a small recombination time and large $v$ (as, for instance, in the case of roughened surfaces), the surface recombination mechanism dominates the PA signal only in the high modulation-frequency range. Consider the example shown in Fig. 7 with, say, $v=300 \mathrm{~cm} / \mathrm{s}$. A sample with $\tau_{\text {eff }}=250 \mu$ s will have the surface recombination process dominating the PA signal only at frequencies greater than $170 \mathrm{~Hz}$, whereas if $\tau_{\text {eff }}=1000 \mu \mathrm{s}$, the surface recombination mechanism dominates at frequencies greater than $69 \mathrm{~Hz}$.

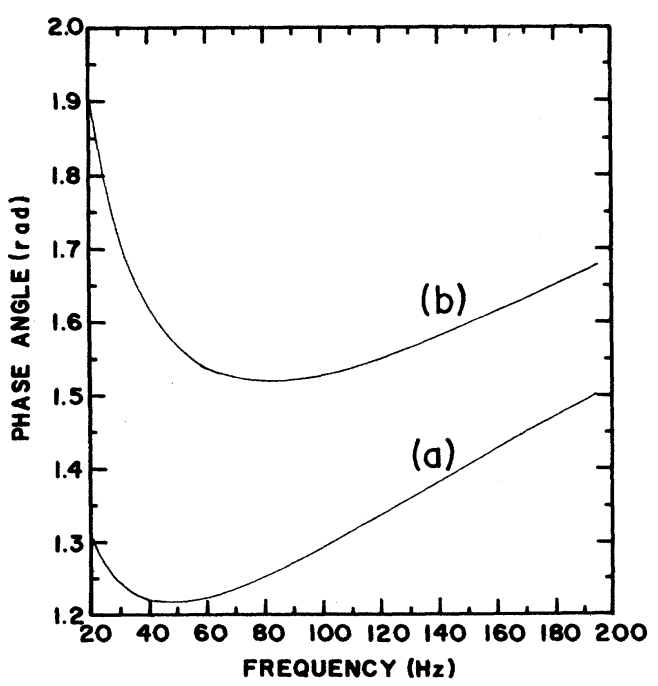

FIG. 6. Variation of the phase angle $\Delta \phi$, given by Eq. (13), as a function of the modulation frequency, for a typical PbTe sample with $\tau_{\text {eff }}=500 \mu$ s for (a) $v=1000 \mathrm{~cm} / \mathrm{s}$ and (b) $v=2000 \mathrm{~cm} / \mathrm{s}$. 


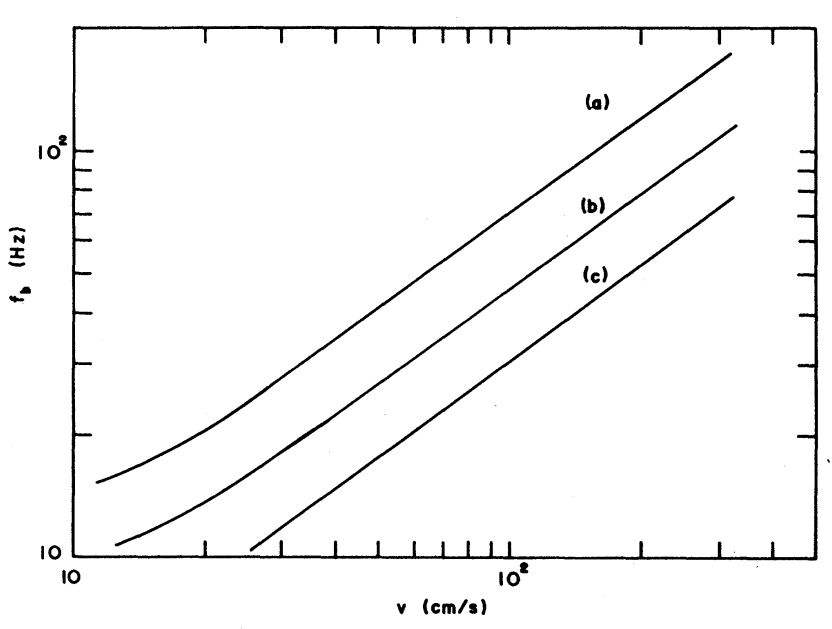

FIG. 7. Breaking frequency vs the surface recombination velocity for a typical PbTe sample with $\tau_{\text {eff }}$ equal to (a) $250 \mu \mathrm{s}$, (b) $500 \mu \mathrm{s}$, and (c) $1000 \mu \mathrm{s}$.

Using Eqs. (12) and (13) for the phase angle together with the values of $D$ and $\alpha_{s}$ for PbTe given in Table I we have carried out the PA phase data fitting for both PbTe samples in the frequency range where the signal is dominated by the nonradiative recombination processes. In Figs. 8 and 9 we show the OPC signal phase angle as a function of the modulation frequency for the RS and PS PbTe samples, respectively. The solid lines in both figures represent the best fit to the data using Eq. (12) for the phase angle, leaving $v$ and $\tau$ as fitting parameters. The results we obtained from the data fitting were $v=2781 \mathrm{~cm} / \mathrm{s}$ and $\tau=0.38 \mu \mathrm{s}$ for the PS sample; and $v=3600 \mathrm{~cm} / \mathrm{s}$ and $\tau=0.38 \mu$ s for the RS sample. In particular, we note that the value of $v$ found for the RS is roughly $29 \%$ larger than that for the PS, while the value

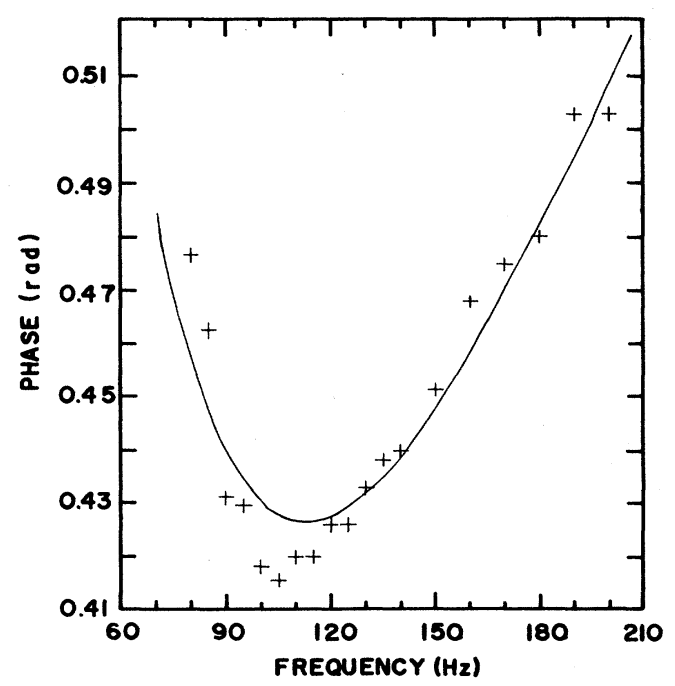

FIG. 8. OPC phase angle for the $298-\mu$ m-thick RS PbTe sample vs the modulation frequency. The solid line represents the data fitting to Eq. (12) of the text.

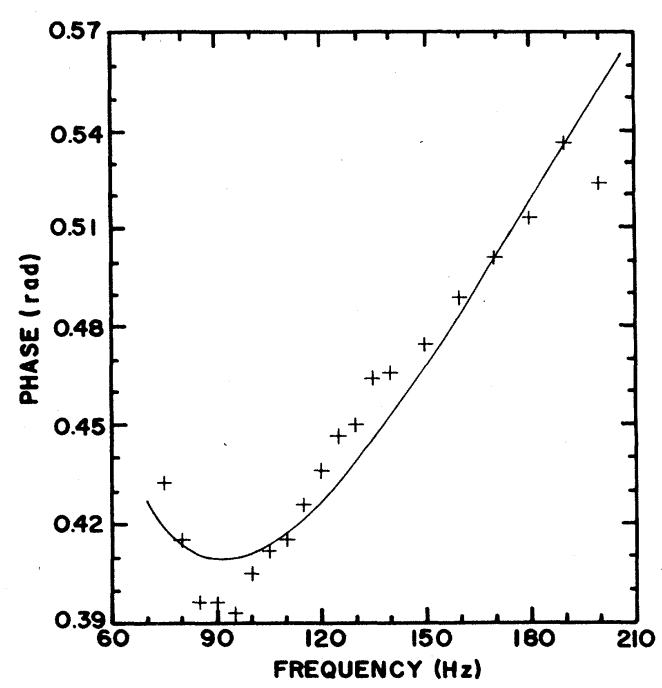

FIG. 9. OPC phase angle for the $320-\mu \mathrm{m}$-thick PS PbTe sample vs the modulation frequency. The solid line represents the data fitting to Eq. (12) of the text.

of $\tau$ remained practically the same for both samples. These results seem physically reasonable in the sense that $\tau$, being essentially a bulk parameter, should be roughly the same for both samples. As for the values of $v$ it is well known in the semiconductor literature that roughened surfaces have larger surface recombination velocities than do polished surfaces, due to the enhanced number of recombination states in the former case. Finally, comparing the phase data shown in Figs. 8 and 9 with the corresponding signal amplitude data shown in Figs. 2 and 3, respectively, we note that the phasebreaking frequency corresponds indeed to the frequency at which the signal amplitude changes its modulation frequency from $f^{-1.5}$ to $f^{-1.0}$, as mentioned above.

\section{CONCLUSIONS}

In this paper we have carried out a modulationfrequency investigation of the PA signal of $\mathrm{PbTe}$ and $\mathrm{Si}$ in the heat-transmission configuration. This was done using both the conventional PA cell as well as the recently proposed OPC geometry. The signal amplitude data clearly showed that the modulation-frequency scanning over the thermally thick region can discriminate the different fast and slow (nonradiative recombination) heat sources responsible for the PA signal. From the phase data dependence on the modulation frequency, in the frequency range where the slow heat sources dominate, it was shown that it is possible to obtain quantitatively the room-temperature values of important semiconductor parameters such as the surface recombination velocity and the nonradiative recombination time. From the experimental point of view this is an interesting result especially for the case of lead salt semiconductors, InSb, and heavily doped semiconductors in general. For these semiconductors the value of $\tau$ at room temperature is particularly difficult to measure since the conventional method (e.g., 
photoconductivity measurements) leads to no results due to the large carrier concentration in these materials at room temperature.

In conclusion, we believe we have demonstrated in this paper an alternative and simple PA method for measuring both surface recombination velocities and nonradiative band-to-band recombination times in semiconductors.
${ }^{1}$ C. C. Ghizoni, M. A. A. Siqueira, H. Vargas, and L. C. M. Miranda, J. Appl. Phys. 32, 554 (1978).

${ }^{2}$ L. Eaves, H. Vargas, and P. J. Williams, Appl. Phys. Lett. 38, 768 (1981).

${ }^{3}$ I. N. Bandeira, H. Closs, and C. C. Ghizoni, J. Photoacoustics 1, 275 (1982).

${ }^{4}$ N. Mikoshiba, H. Nakamura, and K. Tsubouchi, in Proceedings of the IEEE Ultrasonics Symposium, San Diego, 1982 (Institute of Electrical and Electronic Engineers, New York, 1982), p. 580.

${ }^{5}$ V. A. Sablikov and V. B. Sandomirskii, Phys. Status Solidi 120, 471 (1983); Fiz. Tekh. Poluprovodn. 17, 81 (1983) [Sov. Phys.-Semicond. 17, 50 (1983)].

${ }^{6}$ A. N. Vasilev and V. B. Sandomirskii, Fiz. Tekh. Poluprovodn. 18, 1951 (1984) [Sov. Phys. - Semicond. 18, 1095 (1984)]; 18, 1954 (1984) [18, 1221 (1984)].

${ }^{7}$ S. O. Ferreira, C. Ying An, I. N. Bandeira, L. C. M. Miranda, and H. Vargas, Phys. Rev. B 39, 7967 (1989).

${ }^{8}$ H. Vargas and L. C. M. Miranda, Phys. Rep. 161, 43 (1988).

${ }^{9}$ A. Mandelis, Photoacoustic and Thermal Wave Phenomena in Semiconductors (North-Holland, Amsterdam, 1987).
${ }^{10}$ S. Wang, Solid State Electronics (McGraw-Hill, New York, 1966).

${ }^{11}$ M. D. Silva, I. N. Bandeira, and L. C. M. Miranda, J. Phys. E 20, 1476 (1987).

${ }^{12}$ L. F. Perondi and L. C. M. Miranda, J. Appl. Phys. 62, 2955 (1987).

${ }^{13}$ O. Pessoa, Jr., C. L. Cesar, N. A. Patel, H. Vargas, C. C. Ghizoni, and L. C. M. Miranda, J. Appl. Phys. 59, 1316 (1986).

${ }^{14}$ C. Ying An and I. N. Bandeira, Rev. Bras. Fis. Apl. Instr. 1, 52 (1985).

${ }^{15}$ K. Kinoshita and S. Miyazawa, J. Cryst. Growth 57, 141 (1982).

${ }^{16}$ M. Fabbri, I. N. Bandeira, and L. C. M. Miranda, in Proceedings of the 39th Congress of the International Astronautical Federation, Bangalore, India, 1988 (unpublished).

${ }^{17}$ A. Rosenwaig and A. Gersho, J. Appl. Phys. 47, 64 (1976).

${ }^{18} \mathrm{H}$. Vargas and L. C. M. Miranda, in Photoacoustic and Thermal Wave Phenomena in Semiconductors (NorthHolland, Amsterdam, 1987), p. 312. 